Research Article

\title{
Numerical Simulation for General Rosenau-RLW Equation: An Average Linearized Conservative Scheme
}

\author{
Xintian Pan ${ }^{1}$ and Luming Zhang ${ }^{2}$ \\ ${ }^{1}$ School of Mathematics and Information Science, Weifang University, Weifang, Shandong 261061, China \\ 2 Department of Mathematics, Nanjing University of Aeronautics and Astronautics, Nanjing, \\ Jiangsu 210016, China
}

Correspondence should be addressed to Xintian Pan, panxintian@yahoo.com.cn

Received 31 October 2011; Revised 6 February 2012; Accepted 9 March 2012

Academic Editor: John Burns

Copyright (C) 2012 X. Pan and L. Zhang. This is an open access article distributed under the Creative Commons Attribution License, which permits unrestricted use, distribution, and reproduction in any medium, provided the original work is properly cited.

Numerical solutions for the general Rosenau-RLW equation are considered and an energy conservative linearized finite difference scheme is proposed. Existence of the solutions for the difference scheme has been shown. Stability, convergence, and a priori error estimate of the scheme are proved using energy method. Numerical results demonstrate that the scheme is efficient and reliable.

\section{Introduction}

In this paper, we examine the use of the finite difference method for the general Rosenau-RLW equation

$$
u_{t}+u_{x x x x t}-u_{x x t}+u_{x}+\left(u^{p}\right)_{x}=0, \quad x \in\left[x_{l}, x_{r}\right], \quad t \in[0, T]
$$

with an initial condition

$$
u(x, 0)=u_{0}(x), \quad x \in\left[x_{l}, x_{r}\right],
$$


and boundary conditions

$$
u\left(x_{l}, t\right)=u\left(x_{r}, t\right)=0, \quad u_{x x}\left(x_{l}, t\right)=u_{x x}\left(x_{r}, t\right)=0, \quad t \in[0, T],
$$

where $p \geq 2$ is a integer and $u_{0}(x)$ is a known smooth function. When $p=2$, the equation (1.1) is called usual Rosenau-RLW equation. When $p=3,(1.1)$ is called modified Rosenau-RLW equation. tive laws:

It can be proved easily that the problem (1.1)-(1.3) possesses the following conserva-

$$
\begin{gathered}
Q(t)=\int_{x_{l}}^{x_{r}} u(x, t) \mathrm{d} x=\int_{x_{l}}^{x_{r}} u_{0}(x, t) \mathrm{d} x=Q(0), \\
E(t)=\|u\|_{L_{2}}^{2}+\left\|u_{x}\right\|_{L_{2}}^{2}+\left\|u_{x x}\right\|_{L_{2}}^{2}=E(0) .
\end{gathered}
$$

As already pointed out by Fei et al. [1], the nonconservative difference schemes may easily show nonlinear blow-up, and the conservative difference schemes perform better than the non-conservative ones. In [2-15], some conservative finite difference schemes were used for Sine-Gordon equation, Cahn-Hilliard equation, Klein-Gordon equation, a system of Schrödinger equation, Zakharov equations, Rosenau equation, GRLW equation, KleinGordon-Schrödinger equation, respectively. Numerical results of all the schemes are very good.

As far as computational studies are concerned, Zuo et al. [16] have proposed a Crank-Nicolson difference scheme for the Rosenau-RLW equation. The difference scheme in [16] is nonlinear implicit, so it requires heavy iterative calculations and is not suitable for parallel computation. In a recent work [14], we have made some preliminary computation by proposing a conservative linearized difference scheme for GRLW equation which is unconditionally stable and reduces the computational work, and the numerical results are encouraging. In this paper, we continue our work and propose a conservative linearized difference scheme for the general Rosenau-RLW equation which is unconditionally stable and secondorder convergent and simulates conservative laws (1.4)-(1.5) at the same time.

The remainder of this paper is organized as follows. In Section 2, an energy conservative linearized difference scheme for the general Rosenau-RLW equation is described and the

discrete conservative laws of the difference scheme are discussed. In Section 3, we show that the scheme is uniquely solvable. In Section 4, convergence and stability of the scheme are proved. In Section 5, numerical experiments are reported.

\section{An Average Linearized Conservative Scheme and Its Discrete Conservative Law}

In this section, we describe a new conservative difference scheme for the problems of (1.1)(1.3). Let $h$ and $\tau$ be the uniform step size in the spatial and temporal direction, respectively. 
Denote $x_{j}=j h(0 \leq j \leq J), t_{n}=n \tau(0 \leq n \leq N), u_{j}^{n} \approx u\left(x_{j}, t_{n}\right)$ and $Z_{h}^{0}=\left\{u=\left(u_{j}\right) \mid u_{0}=u_{j}=\right.$ $0, j=0,1,2, \ldots, J\}$. Define

$$
\begin{array}{ll}
\left(u_{j}^{n}\right)_{x}=\frac{u_{j+1}^{n}-u_{j}^{n}}{h}, \quad\left(u_{j}^{n}\right)_{\bar{x}}=\frac{u_{j}^{n}-u_{j-1}^{n}}{h}, \quad\left(u_{j}^{n}\right)_{\widehat{x}}=\frac{u_{j+1}^{n}-u_{j-1}^{n}}{2 h}, \\
\left(u_{j}^{n}\right)_{t}=\frac{u_{j}^{n+1}-u_{j}^{n}}{\tau}, \quad\left(u_{j}^{n}\right)_{\hat{t}}=\frac{u_{j}^{n+1}-u_{j}^{n-1}}{2 \tau}, \quad \bar{u}_{j}^{n}=\frac{u_{j}^{n+1}+u_{j}^{n-1}}{2}, \\
\left(u^{n}, v^{n}\right)=h \sum_{j=1}^{J-1} u_{j}^{n} v_{j}^{n}, \quad\left\|u^{n}\right\|^{2}=\left(u^{n}, u^{n}\right), & \left\|u^{n}\right\|_{\infty}=\max _{1 \leq j \leq J-1}\left|u_{j}^{n}\right|,
\end{array}
$$

and in the paper, $C$ denotes a general positive constant which may have different values in different occurrences.

Notice that $\left(u^{p}\right)_{x}=(p /(p+1))\left[u^{p-1} u_{x}+\left(u^{p}\right)_{x}\right]$. We consider the following three-level average linearized conservative scheme for the IBV problems (1.1)-(1.3):

$$
\begin{gathered}
\left(u_{j}^{n}\right)_{\widehat{t}}+\left(u_{j}^{n}\right)_{x x \bar{x} \widehat{x} \hat{t}}-\left(u_{j}^{n}\right)_{x \bar{x} \hat{t}}+\frac{1-\theta}{2}\left(u_{j}^{n+1}+u_{j}^{n-1}\right)_{\widehat{x}}+\theta\left(u_{j}^{n}\right)_{\widehat{x}} \\
+\frac{p}{p+1}\left\{\left(u_{j}^{n}\right)^{p-1}\left(\bar{u}_{j}^{n}\right)_{\widehat{x}}+\left[\left(u_{j}^{n}\right)^{p-1}\left(\bar{u}_{j}^{n}\right)\right]_{\widehat{x}}\right\}=0, \quad 1 \leq j \leq J-1,1 \leq n \leq N-1, \\
u_{j}^{0}=u_{0}\left(x_{j}\right), \quad 1 \leq j \leq J, \\
u_{0}^{n}=u_{J}^{n}=0, \quad\left(u_{0}^{n}\right)_{x \bar{x}}=\left(u_{J}^{n}\right)_{x \bar{x}}=0, \quad 0 \leq n \leq N,
\end{gathered}
$$

where $0 \leq \theta \leq 1$ is a real constant. The scheme (2.2)-(2.4) is three level and linear implicit, so it can be easily implemented. It should be pointed out that we need another suitable twolevel scheme (such as C-N scheme) to compute $u^{1}$. For convenience, the last term of (2.2) is defined by

$$
\Phi\left(u^{n}, \bar{u}^{n}\right)=\frac{p}{p+1}\left\{\left(u_{j}^{n}\right)^{p-1}\left(\bar{u}_{j}^{n}\right)_{\widehat{x}}+\left[\left(u_{j}^{n}\right)^{p-1}\left(\bar{u}_{j}^{n}\right)\right]_{\widehat{x}}\right\}
$$

Lemma 2.1 (see [17]). For any two mesh functions: $u, v \in Z_{h^{\prime}}^{0}$ one has

$$
\begin{gathered}
\left(\left(u_{j}\right)_{x^{\prime}} v_{j}\right)=-\left(u_{j},\left(v_{j}\right)_{\bar{x}}\right), \quad\left(v_{j},\left(u_{j}\right)_{x \bar{x}}\right)=-\left(\left(v_{j}\right)_{x^{\prime}}\left(u_{j}\right)_{x}\right), \\
\left(u_{j},\left(u_{j}\right)_{x \bar{x}}\right)=-\left(\left(u_{j}\right)_{x^{\prime}}\left(u_{j}\right)_{x}\right)=-\left\|u_{x}\right\|^{2} .
\end{gathered}
$$


Furthermore, if $\left(u_{0}^{n}\right)_{x \bar{x}}=\left(u_{J}^{n}\right)_{x \bar{x}}=0$, then

$$
\left(u_{j},\left(u_{j}\right)_{x x \bar{x} \bar{x}}\right)=\left\|u_{x x}\right\|^{2} .
$$

Theorem 2.2. Suppose $u_{0} \in H_{0}^{2}\left[x_{l}, x_{r}\right]$ and $u(x, t) \in C^{5,3}$. Then the scheme (2.2)-(2.4) admits the following invariant:

$$
\begin{aligned}
Q^{n}= & \frac{h}{2} \sum_{j=1}^{J-1}\left(u_{j}^{n+1}+u_{j}^{n}\right)=Q^{n-1}=\cdots=Q^{0}, \\
E^{n}= & \frac{1}{2}\left(\left\|u^{n+1}\right\|^{2}+\left\|u^{n}\right\|^{2}\right)+\frac{1}{2}\left(\left\|u_{x x}^{n+1}\right\|^{2}+\left\|u_{x x}^{n}\right\|^{2}\right) \\
& +\frac{1}{2}\left(\left\|u_{x}^{n+1}\right\|^{2}+\left\|u_{x}^{n}\right\|^{2}\right)+\theta h \tau \sum_{j=1}^{J-1}\left(u_{j}^{n}\right)_{\widehat{x}} u_{j}^{n+1} \\
= & E^{n-1}=\cdots=E^{0} .
\end{aligned}
$$

Proof. Multiplying (2.2) with $h$, according to the boundary conditions (2.4), then summing up for $j$ from 1 to $J-1$, we obtain

$$
\frac{h}{2} \sum_{j=1}^{J-1}\left(u_{j}^{n+1}-u_{j}^{n-1}\right)=0
$$

Let

$$
Q^{n}=\frac{h}{2} \sum_{j=1}^{J-1}\left(u_{j}^{n+1}+u_{j}^{n}\right)
$$

Then we obtain (2.8) from (2.10).

Taking the inner product of (2.2) with $2 \bar{u}^{n}$, according to Lemma 2.1, we have

$$
\begin{gathered}
\frac{1}{2 \tau}\left(\left\|u^{n+1}\right\|^{2}-\left\|u^{n-1}\right\|^{2}\right)+\frac{1}{2 \tau}\left(\left\|u_{x x}^{n+1}\right\|^{2}-\left\|u_{x x}^{n-1}\right\|^{2}\right) \\
+\frac{1}{2 \tau}\left(\left\|u_{x}^{n+1}\right\|^{2}-\left\|u_{x}^{n-1}\right\|^{2}\right)+\theta h \sum_{j=1}^{J-1}\left(u_{j}^{n}\right)_{\widehat{x}} u_{j}^{n+1} \\
-\theta h \sum_{j=1}^{J-1}\left(u_{j}^{n-1}\right)_{\hat{x}} u_{j}^{n}+\left(\Phi\left(u^{n}, \bar{u}^{n}\right), 2 \bar{u}_{j}^{n}\right)=0 .
\end{gathered}
$$


Now, computing the last term of the left-hand side in (2.12), we have

$$
\begin{aligned}
\left(\Phi\left(u^{n}, \bar{u}^{n}\right), 2 \bar{u}_{j}^{n}\right)= & \frac{2 p}{p+1} h \sum_{j=1}^{J-1}\left\{\left(u_{j}^{n}\right)^{p-1}\left(\bar{u}_{j}^{n}\right)_{\widehat{x}}+\left[\left(u_{j}^{n}\right)^{p-1} \bar{u}_{j}^{n}\right]_{\widehat{x}}\right\} \bar{u}_{j}^{n} \\
= & \frac{p}{p+1} \sum_{j=1}^{J-1}\left[\left(u_{j}^{n}\right)^{p-1}\left(\bar{u}_{j+1}^{n}-\bar{u}_{j-1}^{n}\right)+\left(u_{j+1}^{n}\right)^{p-1} \bar{u}_{j+1}^{n}-\left(u_{j-1}^{n}\right)^{p-1} \bar{u}_{j-1}^{n}\right] \bar{u}_{j}^{n} \\
= & \frac{p}{p+1} \sum_{j=1}^{J-1}\left[\left(u_{j}^{n}\right)^{p-1} \bar{u}_{j+1}^{n} \bar{u}_{j}^{n}-\left(u_{j+1}^{n}\right)^{p-1} \bar{u}_{j+1}^{n} \bar{u}_{j}^{n}\right] \\
& -\frac{p}{p+1} \sum_{j=1}^{J-1}\left[\left(u_{j-1}^{n}\right)^{p-1} \bar{u}_{j}^{n} \bar{u}_{j-1}^{n}-\left(u_{j}^{n}\right)^{p-1} \bar{u}_{j}^{n} \bar{u}_{j-1}^{n}\right] \\
= & 0 .
\end{aligned}
$$

Substitute (2.13) into (2.12), and we let

$$
\begin{aligned}
E^{n}= & \frac{1}{2}\left(\left\|u^{n+1}\right\|^{2}+\left\|u^{n}\right\|^{2}\right)+\frac{1}{2}\left(\left\|u_{x x}^{n+1}\right\|^{2}+\left\|u_{x x}^{n}\right\|^{2}\right) \\
& +\frac{1}{2}\left(\left\|u_{x}^{n+1}\right\|^{2}+\left\|u_{x}^{n}\right\|^{2}\right)+\theta h \tau \sum_{j=1}^{J-1}\left(u_{j}^{n}\right)_{\hat{x}} u_{j}^{n+1} .
\end{aligned}
$$

By the definition of $E^{n},(2.9)$ holds.

\section{Solvability}

In this section, we will prove the solvability of the difference scheme (2.2).

Theorem 3.1. The difference scheme (2.2) is uniquely solvable.

Proof. By the mathematical induction. It is obvious that $u^{0}$ is uniquely determined by (2.3). We can choose a second-order method to compute $u^{1}$ (such as C-N scheme [16]). Assuming that $u^{1}, \ldots, u^{n}$ are uniquely solvable, consider $u^{n+1}$ in (2.2) which satisfies

$$
\begin{aligned}
& \frac{1}{2 \tau} u_{j}^{n}+\frac{1}{2 \tau}\left(u_{j}^{n}\right)_{x x \bar{x} \bar{x}}-\frac{1}{2 \tau}\left(u_{j}^{n}\right)_{x \bar{x}}+\frac{1-\theta}{2}\left(u_{j}^{n+1}\right)_{\widehat{x}} \\
& +\frac{p}{2(p+1)}\left\{\left(u_{j}^{n}\right)^{p-1}\left(u_{j}^{n+1}\right)_{\widehat{x}}+\left[\left(u_{j}^{n}\right)^{p-1} u_{j}^{n+1}\right]_{\widehat{x}}\right\}=0 .
\end{aligned}
$$

Taking the inner product of (3.1) with $u^{n+1}$, we obtain

$$
\frac{1}{2 \tau}\left\|u^{n+1}\right\|^{2}+\frac{1}{2 \tau}\left\|u_{x x}^{n+1}\right\|^{2}+\frac{1}{2 \tau}\left\|u_{x}^{n+1}\right\|^{2}+\left(\Psi\left(u^{n}, u^{n+1}\right), u^{n+1}\right)=0,
$$

where $\Psi\left(u^{n}, u^{n+1}\right)=(p / 2(p+1))\left(u_{j}^{n}\right)^{p-1}\left(u_{j}^{n+1}\right)_{\widehat{x}}+\left[\left(u_{j}^{n}\right)^{p-1} u_{j}^{n+1}\right]_{\hat{x}}$. 
Notice that

$$
\begin{aligned}
\left(\Psi\left(u^{n}, u^{n+1}\right), u^{n+1}\right)= & \frac{p h}{2(p+1)} \sum_{j=1}^{J-1}\left\{\left(u_{j}^{n}\right)^{p-1}\left(u_{j}^{n+1}\right)_{\hat{x}}+\left[\left(u_{j}^{n}\right)^{p-1} u_{j}^{n+1}\right]_{\hat{x}}\right\} u_{j}^{n+1} . \\
= & \frac{p}{4(p+1)} \sum_{j=1}^{J-1}\left[\left(u_{j}^{n}\right)^{p-1}\left(u_{j+1}^{n+1}-u_{j-1}^{n+1}\right)\right. \\
& \left.+\left(u_{j+1}^{n}\right)^{p-1} u_{j+1}^{n+1}-\left(u_{j-1}^{n}\right)^{p-1} u_{j-1}^{n+1}\right] u_{j}^{n+1} \\
= & 0 .
\end{aligned}
$$

It follows from (3.2) that

$$
\frac{1}{2 \tau}\left\|u^{n+1}\right\|^{2}+\frac{1}{2 \tau}\left\|u_{x x}^{n+1}\right\|^{2}+\frac{1}{2 \tau}\left\|u_{x}^{n+1}\right\|^{2}=0
$$

That is, there uniquely exists trivial solution satisfying (3.1). Hence, $u_{j}^{n+1}$ in (2.2) is uniquely solvable. This completes the proof of Theorem 3.1.

Remark 3.2. All results above in this paper are correct for IBV problem of the general RosenauRLW equation with finite or infinite boundary.

\section{Convergence and Stability of Finite Difference Scheme}

First we will consider the truncation error of the difference scheme of (2.2)-(2.4). Denote $v_{j}^{n}=u\left(x_{j}, t_{n}\right)$. We define the truncation error as follows:

$$
\begin{aligned}
E r_{j}^{n}= & \left(v_{j}^{n}\right)_{\widehat{t}}+\left(v_{j}^{n}\right)_{x x \overline{x x \hat{t}}}-\left(v_{j}^{n}\right)_{x \widehat{x} \hat{t}}+\frac{1-\theta}{2}\left(v_{j}^{n+1}+v_{j}^{n-1}\right)_{\widehat{x}}+\theta\left(\bar{v}_{j}^{n}\right)_{\hat{x}} \\
& +\frac{p}{p+1}\left\{\left(v_{j}^{n}\right)^{p-1}\left(\bar{v}_{j}^{n}\right)_{\widehat{x}}+\left[\left(v_{j}^{n}\right)^{p-1}\left(\bar{v}_{j}^{n}\right)\right]_{\hat{x}}\right\} .
\end{aligned}
$$

Using Taylor expansion, we obtain that $E r_{j}^{n}=O\left(\tau^{2}+h^{2}\right)$ holds if $\tau, h \rightarrow 0$.

This is that.

Lemma 4.1. Assume $u(x, t)$ is smooth enough, then the local truncation error of difference scheme (2.2) $-(2.4)$ is $O\left(\tau^{2}+h^{2}\right)$.

Next, we will discuss the convergence and stability of finite difference scheme (2.2)(2.4). The following two lemmas are introduced. 
Lemma 4.2 (discrete Sobolev's inequality [18]). There exist two constants $C_{1}$ and $C_{2}$ such that

$$
\left\|u^{n}\right\|_{\infty} \leq C_{1}\left\|u^{n}\right\|+C_{2}\left\|u_{x}^{n}\right\| .
$$

Lemma 4.3 (discrete Gronwall inequality [18]). Suppose $w(k), \rho(k)$ are nonnegative mesh functions and $\rho(k)$ is nondecreasing. If $C>0$ and

$$
w(k) \leq \rho(k)+C \tau \sum_{l=0}^{k-1} w(l), \quad \forall k,
$$

then

$$
w(k) \leq \rho(k) e^{C \tau k}, \quad \forall k
$$

Lemma 4.4. Suppose $u_{0} \in H_{0}^{2}\left[x_{l}, x_{r}\right]$, then the solution $u^{n}$ of (2.2) satisfies $\left\|u^{n}\right\| \leq C,\left\|u_{x}^{n}\right\| \leq C$, which yield $\left\|u^{n}\right\|_{\infty} \leq C(n=1,2, \ldots, N)$.

Proof. It follows from (2.9) that

$$
\begin{aligned}
& \frac{1}{2}\left(\left\|u^{n+1}\right\|^{2}+\left\|u^{n}\right\|^{2}\right)+\frac{1}{2}\left(\left\|u_{x x}^{n+1}\right\|^{2}+\left\|u_{x x}^{n}\right\|^{2}\right)+\frac{1}{2}\left(\left\|u_{x}^{n+1}\right\|^{2}+\left\|u_{x}^{n}\right\|^{2}\right) \\
& \quad=C-\theta h \tau \sum_{j=1}^{J-1}\left(u_{j}^{n}\right)_{\widehat{x}} u_{j}^{n+1} \leq C+\frac{1}{2} \theta \tau\left(\left\|u^{n+1}\right\|^{2}+\left\|u_{x}^{n}\right\|^{2}\right) .
\end{aligned}
$$

Thus

$$
\begin{aligned}
& \frac{1}{2}\left[(1-\theta \tau)\left\|u^{n+1}\right\|^{2}+\left\|u^{n}\right\|^{2}\right]+\frac{1}{2}\left(\left\|u_{x x}^{n+1}\right\|^{2}+\left\|u_{x x}^{n}\right\|^{2}\right) \\
& +\frac{1}{2}\left[(1-\theta \tau)\left\|u_{x}^{n}\right\|^{2}+\left\|u_{x}^{n+1}\right\|^{2}\right] \leq C .
\end{aligned}
$$

This implies for small $\tau$ which satisfies $1-\theta \tau>0$, we get

$$
\left\|u^{n}\right\| \leq C, \quad\left\|u_{x}^{n}\right\| \leq C .
$$

Using Lemma 4.2, we obtain

$$
\left\|u^{n}\right\|_{\infty} \leq C
$$

Remark 4.5. Lemma 4.4 implies that scheme (2.2)-(2.4) is unconditionally stable.

Theorem 4.6. Assume that $u_{0} \in H_{0}^{2}\left[x_{l}, x_{r}\right]$ and $u(x, t) \in C^{5,3}$. Then the solution $u^{n}$ of the scheme (2.2)-(2.4) converges to the solution of problem (1.1)-(1.3) and the rate of convergence is $O\left(\tau^{2}+h^{2}\right)$ by the $\|\cdot\|_{\infty}$ norm. 
Table 1: The errors of numerical solutions at $t=10$ with $p=2$ and $\tau=0.1$.

\begin{tabular}{lcc||cc}
\hline$h$ & $\left\|v^{n}-u^{n}\right\|$ & $\left\|v^{n}-u^{n}\right\|_{\infty}$ & $v \overline{4}-u^{\frac{n}{4}}\|/\| v^{n}-u^{n} \|$ & $\left\|v^{n / 4}-u^{n / 4}\right\|_{\infty} /\left\|v^{n}-u^{n}\right\|_{\infty}$ \\
\hline 0.25 & $1.456039 e-4$ & $1.967455 e-4$ & - & - \\
0.125 & $3.657043 e-5$ & $5.032358 e-5$ & 3.981465 & 3.909609 \\
0.0625 & $9.084201 e-6$ & $1.257422 e-5$ & 4.025718 & 4.002124 \\
0.03125 & $2.202821 e-6$ & $3.052964 e-6$ & 4.123894 & 4.118692 \\
\hline
\end{tabular}

Table 2: The errors of numerical solutions at $t=10$ with $p=4$ and $\tau=0.1$.

\begin{tabular}{lcccc}
\hline$h$ & $\left\|v^{n}-u^{n}\right\|$ & $\left\|v^{n}-u^{n}\right\|_{\infty}$ & $\left\|v^{n / 4}-u^{n / 4}\right\| /\left\|v^{n}-u^{n}\right\|$ & $\left\|v^{n / 4}-u^{n / 4}\right\|_{\infty} /\left\|v^{n}-u^{n}\right\|_{\infty}$ \\
\hline 0.25 & $2.447510 e-4$ & $3.299748 e-4$ & - & - \\
0.125 & $6.146847 e-5$ & $8.525290 e-5$ & 3.981732 & 3.870541 \\
0.0625 & $1.526817 e-5$ & $2.119579 e-5$ & 4.025923 & 4.022161 \\
0.03125 & $3.702240 e-6$ & $5.159101 e-6$ & 4.124036 & 4.108427 \\
\hline
\end{tabular}

Proof. Subtracting (4.1) from (2.2) and letting $e_{j}^{n}=v_{j}^{n}-u_{j}^{n}$, we have

$$
\begin{aligned}
E r_{j}^{n}= & \left(e_{j}^{n}\right)_{\widehat{t}}+\left(e_{j}^{n}\right)_{x x \overline{x x \hat{t}}}-\left(e_{j}^{n}\right)_{x \bar{x} \widehat{t}}+\left(e_{j}^{n}\right)_{\widehat{x}} \\
& +\frac{1-\theta}{2}\left(e_{j}^{n+1}+e_{j}^{n-1}\right)_{\widehat{x}}+\theta\left(e_{j}^{n}\right)_{\widehat{x}} \\
& +\frac{p}{p+1}\left\{\left(v_{j}^{n}\right)^{p-1}\left(\bar{v}_{j}^{n}\right)_{\widehat{x}}+\left[\left(v_{j}^{n}\right)^{p-1} \bar{v}_{j}^{n}\right]_{\hat{x}}\right\} \\
& -\frac{p}{p+1}\left\{\left(u_{j}^{n}\right)^{p-1}\left(\bar{u}_{j}^{n}\right)_{\widehat{x}}+\left[\left(u_{j}^{n}\right)^{p-1} \bar{u}_{j}^{n}\right]_{\hat{x}}\right\} .
\end{aligned}
$$

Taking the inner product in (4.9) with $2 \bar{e}^{n}$, we obtain

$$
\begin{aligned}
\left(E r_{j}^{n}, 2 \bar{e}^{n}\right)= & \frac{1}{2 \tau}\left(\left\|e^{n+1}\right\|^{2}-\left\|e^{n-1}\right\|^{2}\right)+\frac{1}{2 \tau}\left(\left\|e_{x x}^{n+1}\right\|^{2}-\left\|e_{x x}^{n-1}\right\|^{2}\right) \\
& +\frac{1}{2 \tau}\left(\left\|e_{x}^{n+1}\right\|^{2}-\left\|e_{x}^{n-1}\right\|^{2}\right) \\
& +\theta h \sum_{j=1}^{J-1}\left(e_{j}^{n}\right)_{\hat{x}}\left(e_{j}^{n+1}+e_{j}^{n-1}\right)+\left(I+I I, 2 \bar{e}^{n}\right),
\end{aligned}
$$

where

$$
\begin{gathered}
I=\frac{p}{p+1}\left[\left(v_{j}^{n}\right)^{p-1}\left(\bar{v}_{j}^{n}\right)_{\widehat{x}}-\left(u_{j}^{n}\right)^{p-1}\left(\bar{u}_{j}^{n}\right)_{\widehat{x}}\right], \\
I I=\frac{p}{p+1}\left\{\left[\left(v_{j}^{n}\right)^{p-1}\left(\bar{v}_{j}^{n}\right)\right]_{\widehat{x}}-\left[\left(u_{j}^{n}\right)^{p-1} \bar{u}_{j}^{n}\right]_{\widehat{x}}\right\} .
\end{gathered}
$$


Table 3: The errors of numerical solutions at $t=10$ with $p=8$ and $\tau=0.1$.

\begin{tabular}{lcccc}
\hline$h$ & $\left\|v^{n}-u^{n}\right\|$ & $\left\|v^{n}-u^{n}\right\|_{\infty}$ & $\left\|v^{n / 4}-u^{n / 4}\right\| /\left\|v^{n}-u^{n}\right\|$ & $\left\|v^{n / 4}-u^{n / 4}\right\|_{\infty} /\left\|v^{n}-u^{n}\right\|_{\infty}$ \\
\hline 0.25 & $2.854206 e-4$ & $3.856020 e-4$ & - & - \\
0.125 & $7.170514 e-5$ & $9.871671 e-5$ & 3.980476 & 3.906148 \\
0.0625 & $1.781268 e-5$ & $2.465926 e-5$ & 4.025511 & 4.003231 \\
0.03125 & $4.319352 e-6$ & $5.988124 e-6$ & 4.123924 & 4.118027 \\
\hline
\end{tabular}

Table 4: Discrete mass and energy of scheme (2.2) for a few of $\theta$ values at different time $t$ with $h=\tau=0.1$ and $p=2$.

\begin{tabular}{lcccccc}
\hline & \multicolumn{2}{c}{$\theta=0$} & \multicolumn{2}{c}{$\theta=0.5$} & $Q^{n}$ & $\theta=1$ \\
$t$ & $Q^{n}$ & $E^{n}$ & $Q^{n}$ & $E^{n}$ & $E^{n}$ \\
\hline 2 & 3.7953131 & 1.0663504 & 3.7953130 & 1.0661275 & 3.7953129 & 1.0659045 \\
4 & 3.7953035 & 1.0663504 & 3.7953024 & 1.0661275 & 3.7953024 & 1.0659045 \\
6 & 3.7952587 & 1.0663504 & 3.7952598 & 1.0661275 & 3.7952591 & 1.0659045 \\
8 & 3.7950800 & 1.0663504 & 3.7950814 & 1.0661275 & 3.7950829 & 1.0659045 \\
10 & 3.7943581 & 1.0663504 & 3.7943658 & 1.0661275 & 3.7943715 & 1.0659045 \\
\hline
\end{tabular}

According to Lemma 4.4, the fifth term of right-hand side of (4.10) is estimated as follows:

$$
\begin{aligned}
\left(I, 2 \bar{e}^{n}\right) & =\frac{2 p}{p+1} h \sum_{j=1}^{J-1}\left[\left(v_{j}^{n}\right)^{p-1}\left(\bar{v}_{j}^{n}\right)_{\hat{x}}-\left(u_{j}^{n}\right)^{p-1}\left(\bar{u}_{j}^{n}\right)_{\widehat{x}}\right] \bar{e}^{n} \\
& =\frac{2 p}{p+1} h \sum_{j=1}^{J-1}\left(v_{j}^{n}\right)^{p-1}\left(\bar{e}_{j}^{n}\right)_{\hat{x}} \bar{e}^{n}+\frac{2 p}{p+1} h \sum_{j=1}^{J-1}\left[\left(v_{j}^{n}\right)^{p-1}-\left(u_{j}^{n}\right)^{p-1}\right]\left(\bar{u}_{j}^{n}\right)_{\hat{x}} \bar{e}^{n} \\
& =\frac{2 p}{p+1} h \sum_{j=1}^{J-1}\left(v_{j}^{n}\right)^{p-1}\left(\bar{e}_{j}^{n}\right)_{\hat{x}} \bar{e}^{n}+\frac{2 p}{p+1} h \sum_{j=1}^{J-1}\left[e_{j}^{n} \sum_{k=0}^{p-2}\left(v_{j}^{n}\right)^{p-2-k}\left(u_{j}^{n}\right)^{k}\right]\left(\bar{u}_{j}^{n}\right)_{\hat{x}} \bar{e}^{n} \\
& \leq C\left(\left\|\bar{e}_{x}^{n}\right\|^{2}+\left\|e^{n}\right\|^{2}+\left\|\bar{e}^{n}\right\|^{2}\right) \\
& \leq C\left(\left\|e_{x}^{n+1}\right\|^{2}+\left\|e_{x}^{n-1}\right\|^{2}+\left\|e^{n+1}\right\|^{2}+\left\|e^{n}\right\|^{2}+\left\|e^{n-1}\right\|^{2}\right)
\end{aligned}
$$

and similarly we can prove

$$
\left(I I, 2 \bar{e}^{n}\right) \leq C\left(\left\|e_{x}^{n+1}\right\|^{2}+\left\|e_{x}^{n-1}\right\|^{2}+\left\|e^{n+1}\right\|^{2}+\left\|e^{n}\right\|^{2}+\left\|e^{n-1}\right\|^{2}\right)
$$

In addition, it is obvious that

$$
\left(E r_{j}^{n}, 2 \bar{e}^{n}\right) \leq\left\|E r^{n}\right\|^{2}+\frac{1}{2}\left(\left\|e^{n+1}\right\|^{2}+\left\|e^{n-1}\right\|^{2}\right)
$$


Table 5: Discrete mass and energy of scheme (2.2) for a few of $\theta$ values at different time $t$ with $h=\tau=0.1$ and $p=4$.

\begin{tabular}{lcccccc}
\hline & \multicolumn{2}{c}{$\theta=0$} & \multicolumn{2}{c}{$\theta=0.5$} & \multicolumn{2}{c}{$\theta=1$} \\
$t$ & $Q^{n}$ & $E^{n}$ & $Q^{n}$ & $E^{n}$ & $Q^{n}$ & $E^{n}$ \\
\hline 2 & 6.2655606 & 2.8676742 & 6.2655603 & 2.8670879 & 6.2655600 & 2.8665016 \\
4 & 6.2653440 & 2.8676742 & 6.2653437 & 2.8670879 & 6.2653435 & 2.8665016 \\
6 & 6.2648079 & 2.8676742 & 6.2648083 & 2.8670879 & 6.2648086 & 2.8665016 \\
8 & 6.2635545 & 2.8676742 & 6.2635570 & 2.8670879 & 6.2635595 & 2.8665016 \\
10 & 6.2606441 & 2.8676742 & 6.2606529 & 2.8670879 & 6.2606616 & 2.8665016 \\
\hline
\end{tabular}

Table 6: Discrete mass and energy of scheme (2.2) for a few of $\theta$ values at different time $t$ with $h=\tau=0.1$ and $p=8$.

\begin{tabular}{lcccccc}
\hline & \multicolumn{2}{c}{$\theta=0$} & \multicolumn{2}{c}{$\theta=0.5$} & $Q^{n}$ & $Q^{n}$ \\
$t$ & $Q^{n}$ & $E^{n}$ & $Q^{n}$ & $E^{n}$ & $E^{n}$ \\
\hline 2 & 9.7202265 & 4.7351269 & 9.7202105 & 4.7345960 & 9.7201954 & 4.7340650 \\
4 & 9.7140074 & 4.7351270 & 9.7139777 & 4.7345960 & 9.7139486 & 4.7340650 \\
6 & 9.7024346 & 4.7351270 & 9.7023976 & 4.7345960 & 9.7023610 & 4.7340650 \\
8 & 9.6834925 & 4.7351270 & 9.6834566 & 4.7345960 & 9.6834209 & 4.7340650 \\
10 & 9.6536097 & 4.7351270 & 9.6535850 & 4.7345960 & 9.6535602 & 4.7340650 \\
\hline
\end{tabular}

$$
h \sum_{j=1}^{J-1}\left(e_{j}^{n}\right)_{\hat{x}}\left(e_{j}^{n+1}+e_{j}^{n-1}\right) \leq\left\|e_{x}^{n}\right\|^{2}+\frac{1}{2}\left(\left\|e^{n+1}\right\|^{2}+\left\|e^{n-1}\right\|^{2}\right) .
$$

Substituting (4.12)-(4.15) into (4.10), we get

$$
\begin{aligned}
& \frac{1}{2 \tau}\left(\left\|e^{n+1}\right\|^{2}-\left\|e^{n-1}\right\|^{2}\right)+\frac{1}{2 \tau}\left(\left\|e_{x x}^{n+1}\right\|^{2}-\left\|e_{x x}^{n-1}\right\|^{2}\right)+\frac{1}{2 \tau}\left(\left\|e_{x}^{n+1}\right\|^{2}-\left\|e_{x}^{n-1}\right\|^{2}\right) \\
& \leq\left\|E r^{n}\right\|^{2}+\frac{1}{2}\left(\left\|e^{n+1}\right\|^{2}+\left\|e^{n-1}\right\|^{2}\right)+\theta\left[\left\|e_{x}^{n}\right\|^{2}+\frac{1}{2}\left(\left\|e^{n+1}\right\|^{2}+\left\|e^{n-1}\right\|^{2}\right)\right] \\
& +C\left(\left\|e^{n+1}\right\|^{2}+\left\|e^{n-1}\right\|^{2}+\left\|e^{n}\right\|^{2}+\left\|e_{x}^{n+1}\right\|^{2}+\left\|e_{x}^{n}\right\|^{2}+\left\|e_{x}^{n-1}\right\|^{2}\right) .
\end{aligned}
$$

Let $B^{n}=(1 / 2)\left(\left\|e^{n+1}\right\|^{2}+\left\|e^{n}\right\|^{2}\right)+(1 / 2)\left(\left\|e_{x x}^{n+1}\right\|^{2}+\left\|e_{x x}^{n}\right\|^{2}\right)+(1 / 2)\left(\left\|e_{x}^{n+1}\right\|^{2}+\left\|e_{x}^{n}\right\|^{2}\right)$, then $(4.16)$ can be written as follows:

$$
B^{n}-B^{n-1} \leq \tau\left\|E r^{n}\right\|^{2}+C \tau\left(B^{n}+B^{n-1}\right) .
$$

Thus

$$
(1-C \tau)\left(B^{n}-B^{n-1}\right) \leq 2 C \tau B^{n-1}+\tau\left\|E r^{n}\right\|^{2}
$$




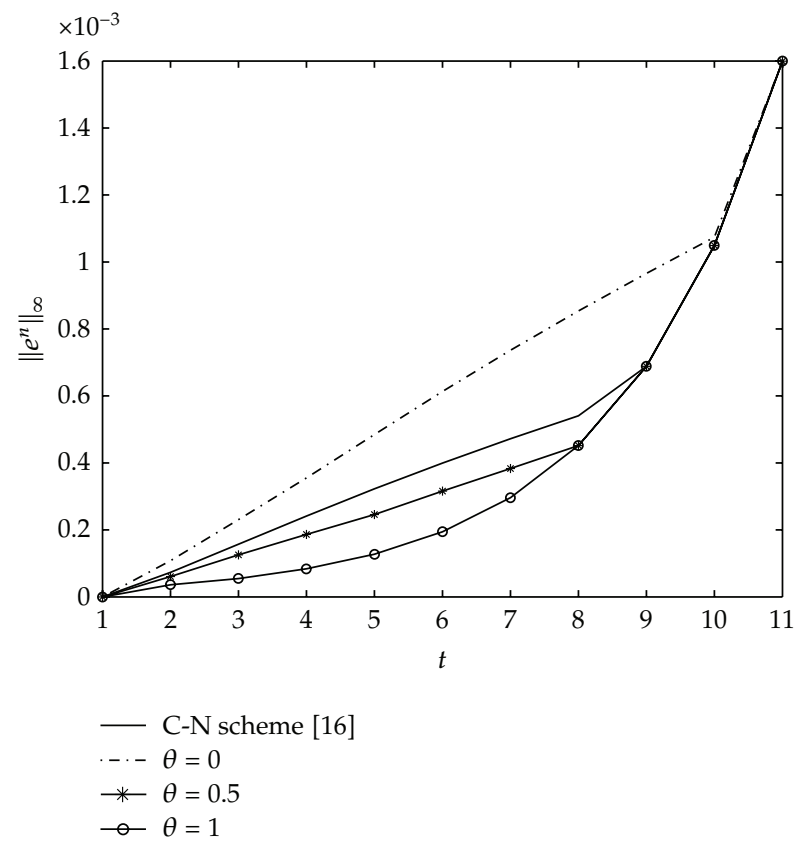

Figure 1: Errors in the sense of $\left\|e^{n}\right\|_{\infty}$ computed by the scheme (2.2) when $h=\tau=0.1$ and $p=4$.

Hence, for $\tau$ sufficiently small, such that $1-C \tau>0$, we obtain

$$
B^{n}-B^{n-1} \leq C \tau B^{n-1}+C \tau\left\|E r^{n}\right\|^{2} .
$$

Summing up (4.19) from 1 to $n$ yields

$$
B^{n} \leq B^{0}+C \tau \sum_{l=1}^{n}\left\|E r^{l}\right\|^{2}+C \tau \sum_{l=1}^{n} B^{l}
$$

Choose a second-order method to compute $u^{1}$ (such as C-N scheme) and notice that

$$
\tau \sum_{l=1}^{n}\left\|E r^{l}\right\|^{2} \leq n \tau \max _{1 \leq l \leq n}\left\|E r^{l}\right\|^{2} \leq T \cdot O\left(\tau^{2}+h^{2}\right)^{2} .
$$

From the discrete initial conditions, we know that $e^{0}$ is of second-order accuracy, then

$$
B^{0}=O\left(\tau^{2}+h^{2}\right)^{2}
$$

Then we obtain

$$
B^{n} \leq O\left(\tau^{2}+h^{2}\right)^{2}+C \tau \sum_{l=0}^{n-1} B^{l}
$$




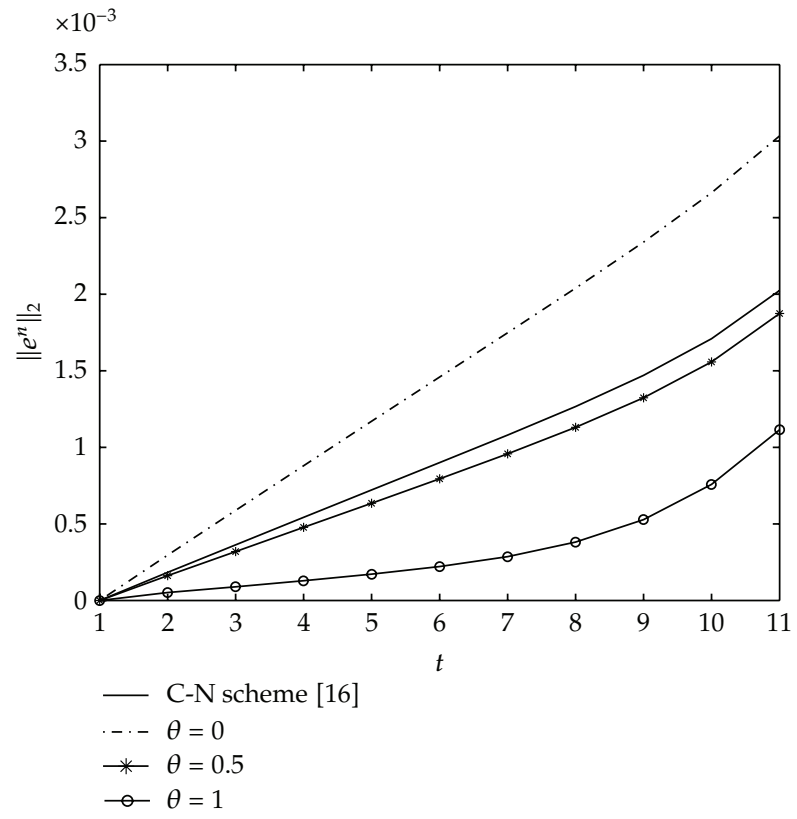

Figure 2: Errors in the sense of $\left\|e^{n}\right\|_{2}$ computed by the scheme (2.2) when $h=\tau=0.1$ and $p=4$.

An application of Lemma 4.3 yields

$$
B^{n} \leq O\left(\tau^{2}+h^{2}\right)^{2}
$$

Thus

$$
\left\|e^{n}\right\| \leq O\left(\tau^{2}+h^{2}\right), \quad\left\|e_{x}^{n}\right\| \leq O\left(\tau^{2}+h^{2}\right), \quad\left\|e_{x x}^{n}\right\| \leq O\left(\tau^{2}+h^{2}\right) .
$$

It follows from Lemma 4.2 that

$$
\left\|e^{n}\right\|_{\infty} \leq O\left(\tau^{2}+h^{2}\right)
$$

This completes the proof of Theorem 4.6.

Similarly, we can prove stability of the difference solution.

Theorem 4.7. Under the conditions of Theorem 4.6, the solution of the scheme (3.1)-(2.4) is unconditionally stable by the $\|\cdot\|_{\infty}$ norm.

\section{Numerical Experiments}

In this section, we conduct some numerical experiments to verify our theoretical results obtained in the previous sections. 


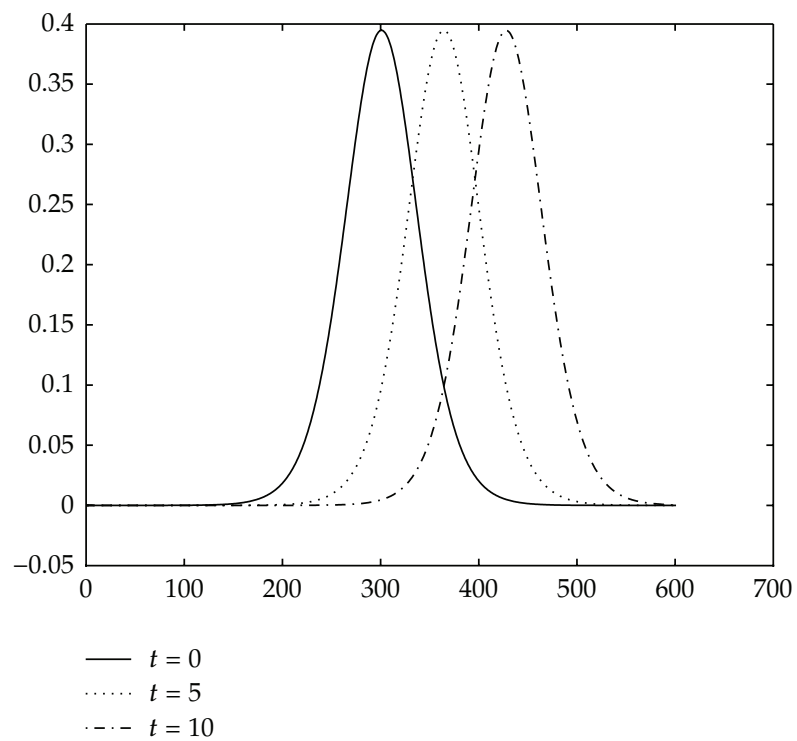

Figure 3: Exact solutions of $u(x, t)$ at $t=0$ and numerical solutions computed by the scheme (2.2) at $t=5$, 10 with $\theta=0$ for $p=2$.

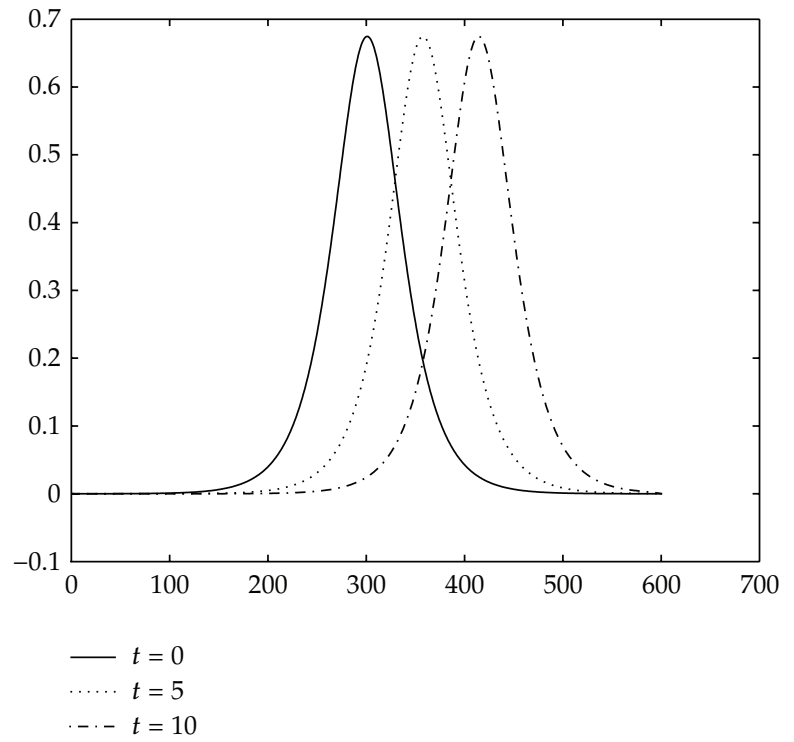

Figure 4: Exact solutions of $u(x, t)$ at $t=0$ and numerical solutions computed by the scheme (2.2) at $t=5$, 10 with $\theta=1$ for $p=4$.

Consider the general Rosenau-RLW equation

$$
u_{t}+u_{x x x x t}-u_{x x t}+u_{x}+\left(u^{p}\right)_{x}=0, \quad x \in\left[x_{l}, x_{r}\right], \quad t \in[0, T]
$$

with an initial condition

$$
u(x, 0)=u_{0}(x), \quad x \in\left[x_{l}, x_{r}\right]
$$


and boundary conditions

$$
u\left(x_{l}, t\right)=u\left(x_{r}, t\right)=0, \quad u_{x x}\left(x_{l}, t\right)=u_{x x}\left(x_{r}, t\right)=0, \quad t \in[0, T] .
$$

The exact solution of the system (5.1)-(5.2) has the following form:

$$
u(x, t)=e^{\ln \left\{(p+3)(3 p+1)(p+1) /\left[2\left(p^{2}+3\right)\left(p^{2}+4 p+7\right)\right]\right\} /(p-1)} \operatorname{sech}^{4 /(p-1)}\left[\frac{p-1}{\sqrt{4 p^{2}+8 p+20}}(x-c t)\right]
$$

where $p \geq 2$ is a integer and $c=\left(p^{4}+4 p^{3}+14 p^{2}+20 p+25\right) /\left(p^{4}+4 p^{3}+10 p^{2}+12 p+21\right)$.

It follows from (5.4) that the initial-boundary value problem (5.1)-(5.3) is consistent to the initial value problem (5.1)-(5.2) for $-x_{l} \gg 0, x_{r} \gg 0$. In the numerical experiments, we take $-x_{l}=x_{r}=30, T=10$, and consider three cases $p=2,4,8$, respectively. The errors in the sense of $L_{\infty}$-norm and $L_{2}$-norm of the numerical solutions are listed on Tables 1, 2, and 3 for three cases $p=2,4,8$ with $\theta=1$. Tables 1,2 , and 3 verify the second-order convergence and good stability of the numerical solutions.

We have shown in Theorem 2.2 that the numerical solution $u^{n}$ of the scheme (2.2) satisfies the conservation of discrete mass and energy, respectively. In Tables 4, 5, and 6, the values of $(h / 2) \sum_{j=1}^{J-1}\left(u_{j}^{n+1}+u_{j}^{n}\right)$ and $(1 / 2)\left(\left\|u^{n+1}\right\|^{2}+\left\|u^{n}\right\|^{2}\right)+(1 / 2)\left(\left\|u_{x x}^{n+1}\right\|^{2}+\left\|u_{x x}^{n}\right\|^{2}\right)+$ $(1 / 2)\left(\left\|u_{x}^{n+1}\right\|^{2}+\left\|u_{x}^{n}\right\|^{2}\right)+\theta h \tau \sum_{j=1}^{J-1}\left(u_{j}^{n}\right)_{\widehat{x}} u_{j}^{n+1}$ for the scheme (2.2) are presented for three cases $p=2,4,8$ under steps $h=\tau=0.1$ with $\theta=0,0.5$ and 1 , respectively. It is easy to see from Tables 4,5 , and 6 that the scheme (2.2) preserves the discrete mass and discrete energy very well; thus it can be used to computing for a long time.

We make a comparison between C-N scheme [16] and our scheme with $\theta=0,0.5,1$ under the meshes $h=\tau=0.1$ in Figures 1 and 2 when $p=4$. It is obvious from Figures 1 and 2 that our scheme performs better than $\mathrm{C}-\mathrm{N}$ scheme [16] in the numerical precision when $\theta=0.5$ and 1 . Figures 1 and 2 also show that numerical precision of the scheme (2.2) depends on the choice of parameter $\theta$. The curves of the solitary waves with time computed by the scheme (2.2) with $\theta=0$ for $p=2$ and $\theta=1$ for $p=4$ under mesh sizes of $h=\tau=0.1$ are given in Figures 3 and 4, respectively; the waves at $t=5,10$ agree with the ones at $t=0$ quite well, which also demonstrate the accuracy of the scheme in present paper.

From the numerical results, the scheme of this paper is accurate and efficient.

\section{Acknowledgments}

This work is supported by the Youth Research Foundation of WFU (no. 2011Z17). The authors would like to thank the editor and the reviewers for their valuable comments and suggestions.

\section{References}

[1] Z. Fei, V. M. Pérez-García, and L. Vazquez, "Numerical simulation of nonlinear Schrodinger systems: a new conservative scheme," Applied Mathematics and Computation, vol. 71, no. 2-3, pp. 165-177, 1995.

[2] Z. Fei and L. Vazquez, "Two energy conserving numerical schemes for the sine-Gordon equation," Applied Mathematics and Computation, vol. 45, no. 1, pp. 17-30, 1991. 
[3] S. M. Choo and S. K. Chung, "Conservative nonlinear difference scheme for the Cahn-Hilliard equation," Computers $\mathcal{E}$ Mathematics with Applications, vol. 36, no. 7, pp. 31-39, 1998.

[4] S. M. Choo, S. K. Chung, and K. I. Kim, "Conservative nonlinear difference scheme for the CahnHilliard equation. II," Computers \& Mathematics with Applications, vol. 39, no. 1-2, pp. 229-243, 2000.

[5] Y. S. Wong, Q. Chang, and L. Gong, "An initial-boundary value problem of a nonlinear Klein-Gordon equation," Applied Mathematics and Computation, vol. 84, no. 1, pp. 77-93, 1997.

[6] T.-C. Wang and L.-M. Zhang, "Analysis of some new conservative schemes for nonlinear Schrodinger equation with wave operator," Applied Mathematics and Computation, vol. 182, no. 2, pp. 1780-1794, 2006.

[7] T. Wang, B. Guo, and L. Zhang, "New conservative difference schemes for a coupled nonlinear Schrodinger system," Applied Mathematics and Computation, vol. 217, no. 4, pp. 1604-1619, 2010.

[8] Q. Chang, E. Jia, and W. Sun, "Difference schemes for solving the generalized nonlinear Schrodinger equation," Journal of Computational Physics, vol. 148, no. 2, pp. 397-415, 1999.

[9] Q. S. Chang, B. L. Guo, and H. Jiang, "Finite difference method for generalized Zakharov equations," Mathematics of Computation, vol. 64, no. 210, pp. 537-553, 1995.

[10] Q. S. Chang and H. Jiang, "A conservative difference scheme for the Zakharov equations," Journal of Computational Physics, vol. 113, no. 2, pp. 309-319, 1994.

[11] R. T. Glassey, "Convergence of an energy-preserving scheme for the Zakharov equations in one space dimension," Mathematics of Computation, vol. 58, no. 197, pp. 83-102, 1992.

[12] J. Hu and K. Zheng, "Two conservative difference schemes for the generalized Rosenau equation," Boundary Value Problems, vol. 2010, Article ID 543503, 18 pages, 2010.

[13] K. Omrani, F. Abidi, T. Achouri, and N. Khiari, "A new conservative finite difference scheme for the Rosenau equation," Applied Mathematics and Computation, vol. 201, no. 1-2, pp. 35-43, 2008.

[14] L. Zhang, "A finite difference scheme for generalized regularized long-wave equation," Applied Mathematics and Computation, vol. 168, no. 2, pp. 962-972, 2005.

[15] L. Zhang, "Convergence of a conservative difference scheme for a class of Klein-Gordon-Schrodinger equations in one space dimension," Applied Mathematics and Computation, vol. 163, no. 1, pp. 343-355, 2005.

[16] J.-M. Zuo, Y.-M. Zhang, T.-D. Zhang, and F. Chang, "A new conservative difference scheme for the general Rosenau-RLW equation," Boundary Value Problems, vol. 2010, Article ID 516260, 13 pages, 2010.

[17] B. Hu, Y. Xu, and J. Hu, "Crank-Nicolson finite difference scheme for the Rosenau-Burgers equation," Applied Mathematics and Computation, vol. 204, no. 1, pp. 311-316, 2008.

[18] Y. L. Zhou, Applications of Discrete Functional Analysis to the Finite Difference Method, International Academic Publishers, Beijing, China, 1991. 


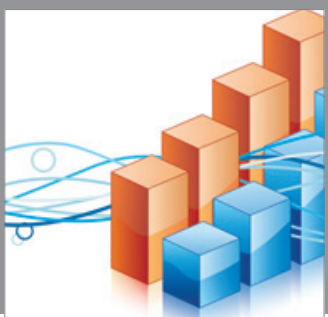

Advances in

Operations Research

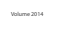

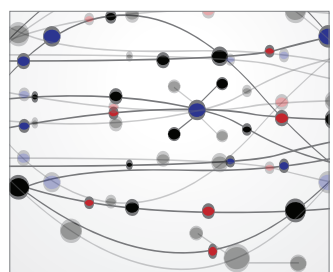

\section{The Scientific} World Journal
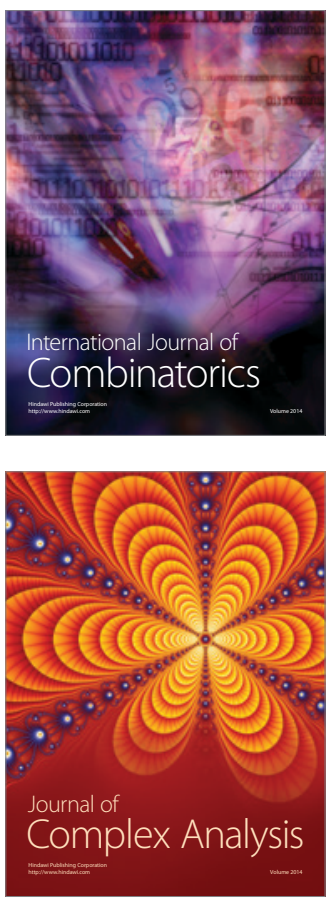

International Journal of

Mathematics and

Mathematical

Sciences
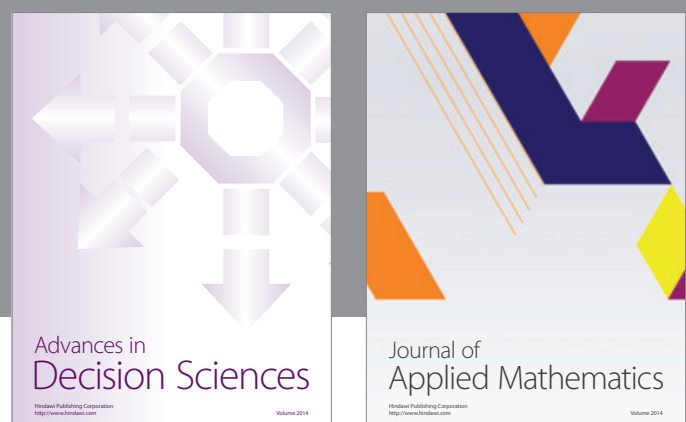

Journal of

Applied Mathematics
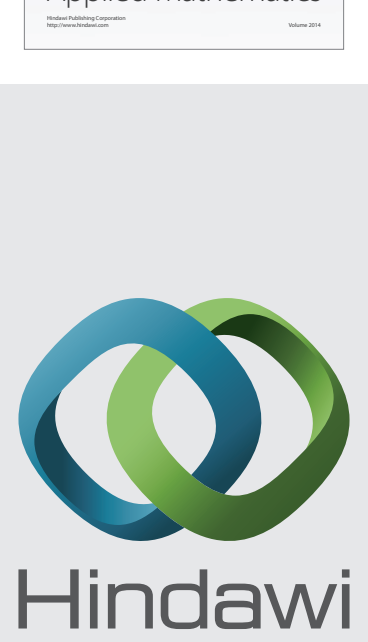

Submit your manuscripts at http://www.hindawi.com
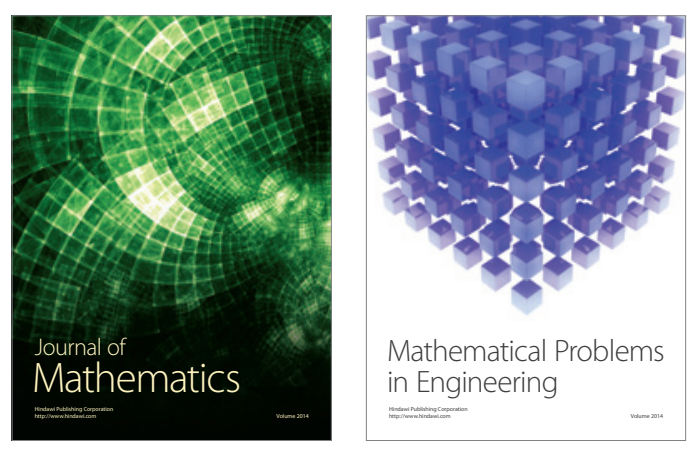

Mathematical Problems in Engineering
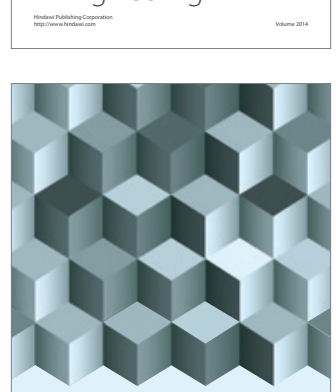

Journal of

Function Spaces
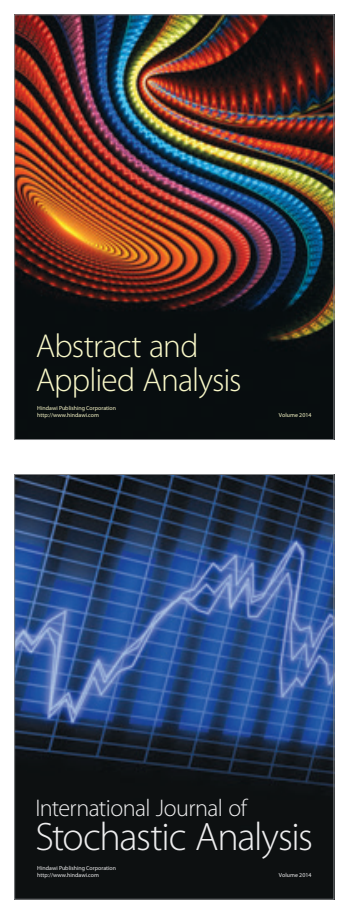

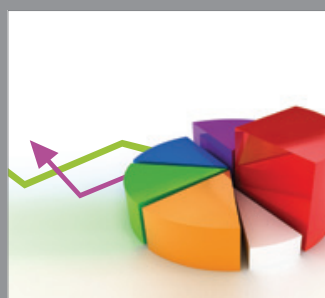

ournal of

Probability and Statistics

Promensencen
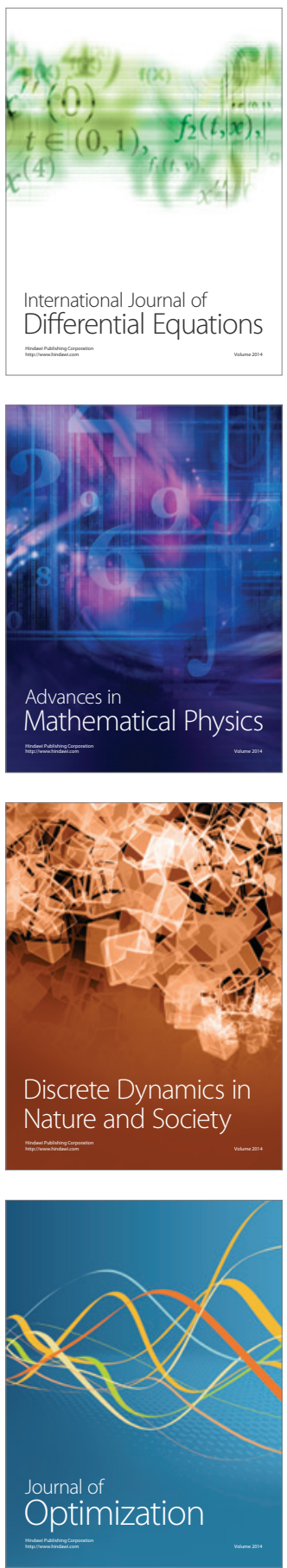